\title{
A NEW APPROACH IN DECOMPOSITION OVER MULTIPLE-OVERCOMPLETE DICTIONARIES WITH APPLICATION TO IMAGE INPAINTING
}

\author{
SeyyedMajid Valiollahzadeh ${ }^{(a) *}$, Mohammad Nazari $^{(b)}$, Massoud Babaie-Zadeh $^{(a)}$, Christian Jutten $^{(c)}$ \\ ${ }^{(a)}$ Department of Electrical Engineering, Sharif University of Technology, Tehran, Iran \\ ${ }^{(b)}$ Department of Electrical Engineering, Amirkabir University of Technology, Tehran, Iran \\ ${ }^{(c)}$ GIPSA-lab, Grenoble, France and Institut Universitaire de France
}

\begin{abstract}
Recently, great attention was intended toward overcomplete dictionaries and the sparse representations they can provide. In a wide variety of signal processing problems, sparsity serves a crucial property leading to high performance. Decomposition of a given signal over two or more dictionaries with sparse coefficients is investigated in this paper. This kind of decomposition is useful in many applications such as inpainting, denoising, demosaicing, speech source separation, high-quality zooming and so on. This paper addresses a novel technique of such a decomposition and investigates this idea through inpainting of images which is the process of reconstructing lost or deteriorated parts of images or videos. When samples are missed in an image, the the original sparsity level in representing coefficients is changed, so with an iterative method we can estimate the original level. Simulations are presented to demonstrate the validation of our approach.
\end{abstract}

\section{INTRODUCTION}

Sparse signal decomposition of signals on an overcomplete dictionary was of great interest among researchers in past few years and serve many interesting applications [1]. The main assumption over these signals is that they are linear mixtures of building atoms and also only few of these atoms will participate in the reconstruction. In the context of image processing an interesting decomposition application would be separating texture from non-texture part to be used in areas from compression to analysis and synthesis of an image [2][3]. Inpainting consists in problems like filling the holes, reconstructing lost or deteriorated parts of images or videos, removal of scratches in old photos, removal of unwanted text or graphic and is an interesting inverse problem with lots of research momentum in recent years [4] dealing

\footnotetext{
* This work has been partially supported by Iran National Science Foundation (INSF) under contract number $86 / 994$, by Iran Telecommunications Research Center (ITRC), and also by ISMO and French embassy in Iran in the framework of a Gundi-Shapour collaboration program.
}

highly with such decomposition. Pioneered by the work of Sapiro et. al. [5], total variation was used in this respect taking mainly the geometrical contents into consideration. Since images contain both geometrical and textural information, decomposition should be done in two layers.

This approach has been presented in [6]-[7] and the layers to which an image is decomposed are called texture and cartoon. The inpainting process is done in each layer separately and afterwards the output will be formed by summing up these layers. The crucial part in this approach is layer decomposition and will extend the notions of total variations. By this trend, if any failure in the inpainting of each layer is presented, superimposing of two layers will lead in less visual artifact and hence quite satisfactory results.

In some recent works sparsity was taken into account as additional criteria to decompose an image to these layers. To this end, we need two dictionaries, two mutually incoherent, one to represent the texture and the other for the cartoon. Both should provide the sparse representation for the corresponding layer image while yielding nonsparse for the other. Combination of these two dictionaries into one and performing the BPDN (Basis Pursuit denoising) [1] algorithm seeking the sparsest solution has shown to perform well and even can be improved by further applying the totalvariation regularization.

Elad et. al. [8, 9] proposed an inpainting algorithm capable of filling in holes in either texture or cartoon content, or any combination thereof extending employment of separation by sparsity, so that the missing samples fit naturally into the layer separation framework. The main advantageous point of this approach is the global processing trend toward the image rather the local one. Also it deploys general overcomplete dictionaries which can be better established for a typical image content.

What is presented in this paper is finding a suitable decomposition of an image over two dictionaries on the basis of sparse representations as a modeling of the overall problem of inpainting. One of decomposed images serves as cartoon and the other as texture with this knowledge that the contents of the cartoon is piecewise smooth (differen- 
tiable). Inspired by the work of Mohimani, et. al. [10] for finding the sparsest solution of an Underdetermined System of Linear Equations (USLE) through the smoothed $\ell^{0}$-norm, we extend this approach in two dimensional models to solve this prior modeling. The outline of the paper is as follows. In section 2, we briefly present the modeling scenario to decompose a signal over two incoherent dictionaries. In section 3 we model the inpainting problem and present the final algorithm. We discuss some simulation results to validate the proposed algorithm in section 4 and finally conclusion and summary of later work are discussed in the last section.

\section{IMAGE DECOMPOSITION}

Let the input image c containing $N$ total pixels, be presented as a one-dimensional vector. This image is to be decomposed over two distinct dictionaries, $\mathbf{A}$ and $\mathbf{B}$ the former corresponding to texture and the latter to cartoon. Both provide sparse representation for the image of their kind and non-sparse for the other, written formally as:

$$
\begin{array}{ll}
\mathbf{c}_{1}=\mathbf{A} \mathbf{s}_{1}, & \mathbf{s}_{1} \text { is sparse } \\
\mathbf{c}_{2}=\mathbf{B} \mathbf{s}_{2}, & \mathbf{s}_{2} \text { is sparse }
\end{array}
$$

where $\mathbf{c}_{1}$ corresponds to the first decomposed image (cartoon) and $\mathbf{c}_{2}$ corresponds to the other one (texture). Sparsity of a vector $\mathbf{s}$ is quantified by its $\ell^{0}$-norm, denoted by $\|\mathbf{s}\|_{0}$, which is the number of its nonzero elements. There are two assumptions over these dictionaries [8,9]: Firstly, these two dictionaries should be incoherent, i.e. the texture dictionary is not able to represent the cartoon image sparsely and vice versa. Secondly, the dictionary assigned to texture should be such that if the texture appears in local parts of the image and is zero elsewhere, representation is still sparse, implying somehow that it should employ a multiscale and local analysis of the image content. Now, we seek a sparse representation over the combined dictionary:

$$
\left\{\mathbf{s}_{1}, \mathbf{s}_{2}\right\}=\underset{\mathbf{s}_{1}, \mathbf{s}_{2}}{\operatorname{argmin}}\left\{\left\|\mathbf{s}_{1}\right\|_{0}+\left\|\mathbf{s}_{2}\right\|_{0}\right\} \text { s.t. } \mathbf{c}=\mathbf{A} \mathbf{s}_{1}+\mathbf{B} \mathbf{s}_{2}
$$

The problem is non-convex and seemingly intractable due to combinatorial search it needs, however inspired by the work of Mohimani et. al. [10], we can find $\mathbf{s}_{1}, \mathbf{s}_{2}$ as it using smoothed $\ell^{0}$-norm. Smoothed $\ell^{0}$-norm of a vector is an approximation to its norm and is defined as:

$$
\|\boldsymbol{\alpha}\|_{0} \simeq m-F_{\sigma}(\boldsymbol{\alpha})=m-\sum_{i} \exp \left(-\alpha_{i}^{2} / 2 \sigma^{2}\right)
$$

where $\sigma$ is a parameter determining a tradeoff between the accuracy of approximation and the smoothness of $F_{\sigma}(\boldsymbol{\alpha})$. Minimizing the $\ell^{0}$-norm of subject to $\mathbf{b}=\boldsymbol{\Phi} \boldsymbol{\alpha}$ requires then to maximize $F_{\sigma}(\boldsymbol{\alpha})$ for a small value of $\sigma$. For a small $\sigma$, $F_{\sigma}(\boldsymbol{\alpha})$ is highly non-smooth with lots of local maxima. To overcome this difficulty [10] suggests to use a decreasing sequence of $\sigma$. The algorithm initially starts with minimum $\ell^{2}$-norm solution of $\mathbf{b}=\mathbf{\Phi} \boldsymbol{\alpha}$, which corresponds to the maximizer of $F_{\sigma}(\boldsymbol{\alpha})$ when $\sigma \rightarrow \infty$.

Using a similar idea, we want to minimize a cost function $J_{\sigma}(\mathbf{s})$-which will be introduced in the next sectionsubject to $\mathbf{c}=\mathbf{A} \mathbf{s}_{1}+\mathbf{B s}_{2}$. The minimization should be done for small $\sigma$ and in order to avoid trapping into local minima we use a decreasing sequence of $\left[\sigma_{1} \sigma_{2} \ldots \sigma_{J}\right]$ and then minimize $J_{\sigma}(\mathbf{s})$ for each $\sigma$, with the starting point yielded by the maximizer of the previous (larger) $\sigma$. Moreover the process is initialized by:

$$
\begin{array}{r}
{\left[\begin{array}{ll}
\mathbf{A} & \mathbf{B}
\end{array}\right]\left[\begin{array}{c}
\mathbf{s}_{1} \\
\mathbf{s}_{2}
\end{array}\right]=\mathbf{c} \quad \Longrightarrow} \\
{\left[\begin{array}{l}
\mathbf{s}_{1} \\
\mathbf{s}_{2}
\end{array}\right]=\left[\begin{array}{ll}
\mathbf{A} & \mathbf{B}
\end{array}\right]^{\dagger} \mathbf{c}=\left[\begin{array}{c}
\left(\mathbf{P}_{\mathbf{B}}^{\perp} \mathbf{A}\right)^{\dagger} \\
\left(\mathbf{P}_{\mathbf{A}}^{\perp} \mathbf{B}\right)^{\dagger}
\end{array}\right] \mathbf{c}}
\end{array}
$$

where $\left(\mathbf{P}_{\mathbf{B}}^{\perp} \mathbf{A}\right)^{\dagger}$ and $\left(\mathbf{P}_{\mathbf{A}}^{\perp} \mathbf{B}\right)^{\dagger}$ are the orthogonal projections of the corresponding matrices:

$$
\begin{array}{r}
\mathbf{P}_{\mathbf{A}}^{\perp}=\mathbf{I}-\mathbf{A}^{T}\left(\mathbf{A A}^{T}\right)^{-1} \mathbf{A} \\
\mathbf{P}_{\mathbf{B}}^{\perp}=\mathbf{I}-\mathbf{B}^{T}\left(\mathbf{B B}^{T}\right)^{-1} \mathbf{B}
\end{array}
$$

Then we use $L$ iterations of the steepest ascent algorithm, followed by a projection onto the feasible set which is:

$$
\left[\begin{array}{l}
\mathbf{s}_{1} \\
\mathbf{s}_{2}
\end{array}\right] \longleftarrow\left[\begin{array}{l}
\mathbf{s}_{1} \\
\mathbf{s}_{2}
\end{array}\right]-\left[\begin{array}{ll}
\mathbf{A} & \mathbf{B}
\end{array}\right]^{\dagger}\left(\mathbf{c}-\mathbf{A} \mathbf{s}_{1}-\mathbf{B} \mathbf{s}_{2}\right)
$$

For more explanations about choosing the sequence see [10].

\section{MODELING INPAINTING AND THE FINAL PROPOSED ALGORITHM}

Suppose the original image is multiplied with a binary mask matrix of $\mathbf{M}$ and the main aim is to fill out properly the missing parts of the image behind the mask. The corrupted available image is to be decomposed in two images called texture and cartoon and then each layer is tried to fill out, simultaneously. All the information available is that the location of the missing samples in the original image is known and components of the image can be represented sparsely over their known dictionaries. This way we want to have an estimate of decomposition of the image $\mathbf{A} \mathbf{s}_{1}$ and $\mathbf{B} \mathbf{s}_{2}$ (texture and cartoon). The fidelity of the representation is measured with respect to the only existing measurements and missing samples are not taken into account. The total-variation penalty, suppresses the typical ringing artifacts encountered in using linear transforms. This point is highlighted near sharp edges where ringing artifacts are noticeably visible. 


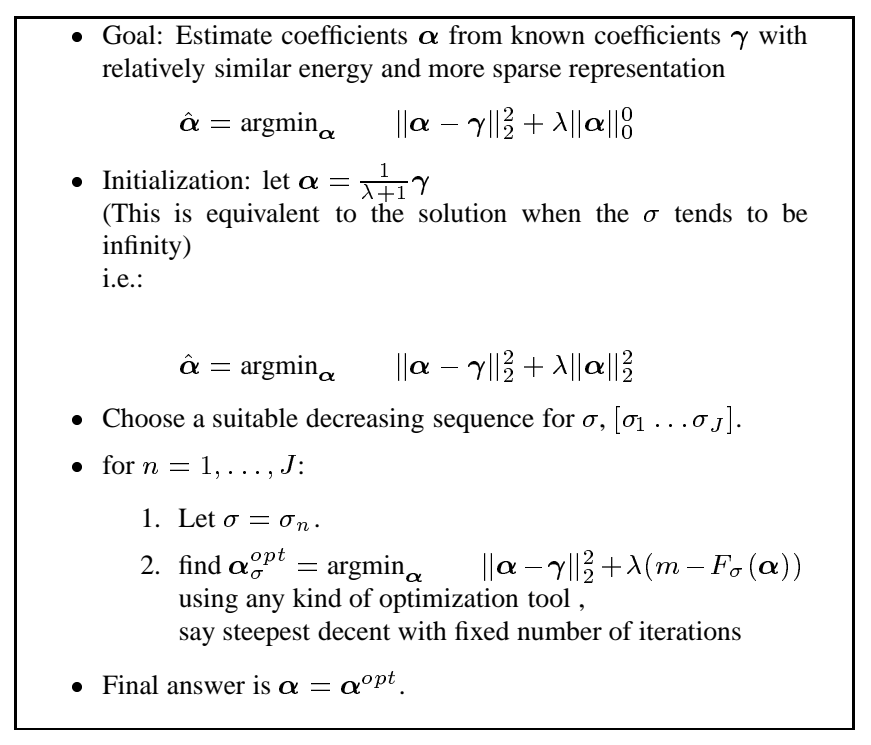

Fig. 1. Algorithm for estimating coefficients.

Suppose that missing pixels of the image are masked with a diagonal mask matrix $\mathbf{M}$ (of which has value ' 1 ' over the existing pixels and ' 0 ' over the missing pixels) we propose restoring the image by optimizing the following problem:

$$
\begin{array}{r}
\left\{\mathbf{s}_{1}^{\text {opt }}, \mathbf{s}_{2}^{\text {opt }}\right\}=\underset{\mathbf{s}_{1}, \mathbf{s}_{2}}{\operatorname{argmin}}\left\|\mathbf{s}_{1}\right\|_{0}+\left\|\mathbf{s}_{2}\right\|_{0}+ \\
\lambda\left\|\mathbf{M}\left(\mathbf{c}-\mathbf{A} \mathbf{s}_{1}-\mathbf{B} \mathbf{s}_{2}\right)\right\|_{2}^{2}+\mu \mathbf{T V}\left(\mathbf{A} \mathbf{s}_{1}\right)
\end{array}
$$

in which we have $\mathbf{T V}(\mathbf{s})=\|\nabla \mathbf{s}\|_{1}$. Here the total variation of an image $\mathbf{T V}(\mathbf{I})$ is essentially $\ell^{1}$-norm of the gradient. Considering this term we force to have a sparser gradient and hence more piecewise smooth image. Having found the optimum coefficients, the recovered image would be:

$$
\mathbf{c}=\mathbf{A} \mathbf{s}_{1}^{o p t}+\mathbf{B s}_{2}^{o p t}
$$

These coefficients to be found can be relaxed as stated in the previous part:

$$
\begin{array}{r}
J_{\sigma}=\left(m_{1}-F_{\sigma}\left(\mathbf{s}_{1}\right)\right)+\left(m_{2}-F_{\sigma}\left(\mathbf{s}_{2}\right)\right) \\
+\lambda\left\|\mathbf{M}\left(\mathbf{c}-\mathbf{A} \mathbf{s}_{1}-\mathbf{B s}_{2}\right)\right\|_{2}^{2}+\mu \mathbf{T V}\left(\mathbf{A} \mathbf{s}_{1}\right)
\end{array}
$$

where $m_{1}$ and $m_{2}$ are the length of $\mathbf{s}_{1}, \mathbf{s}_{2}$ coefficients, not necessarily equivalent. Solving (10) leads to a better inpainting method in comparison with work presented in [9] due to this fact that general overcomplete representation has taken into the account. This allows to better match natural image content by choosing the transform to strengthen the sparsity assumption, which is at the heart of these methods. The algorithm we use to solve this optimization is basically on the basis of block-coordinate-relaxation method $[8,12]$ taking the advantage of $\ell^{0}$-norm solution [13]. The overall algorithm is as follows: similar to block-coordinate descent[12], one image (say $\mathbf{c}_{1}$ ) is fixed while the other, $\mathbf{c}_{2}$ is updated and vice-versa. Consider the case we want we want to update $\mathbf{c}_{1}$ and hence the $\mathbf{c}_{2}$ is updated. The residual image $\mathbf{R}=\mathbf{M}\left(\mathbf{c}-\mathbf{c}_{1}-\mathbf{c}_{2}\right)$ is computed in which missing pixels are forced to be zero. $\mathbf{R}+\mathbf{c}_{1}$ (simply one part of the image plus the residue), which obviously does not contain any holes, is transformed into its corresponding sparse space (curvelet coefficients for $\mathbf{c}_{1}$ and thus the coefficients $\gamma$ is yielded. Since so far we assumed that $\mathbf{c}_{1}$ has the sparsest coefficients over the dictionary of $\mathbf{A}$, missing some samples will affect this sparsity. In order to retrieve the coefficients of the true coefficients of the representing $\mathbf{c}_{1}$, one estimation could be:

$$
\hat{\boldsymbol{\alpha}}=\operatorname{argmin}_{\boldsymbol{\alpha}} \quad\|\boldsymbol{\alpha}-\gamma\|_{2}^{2}+\lambda\|\boldsymbol{\alpha}\|_{0}^{0}
$$

$\hat{\boldsymbol{\alpha}}$ can be interpreted in this way that it is the closest possible coefficient set to $\boldsymbol{\alpha}$ in energy but very more sparsely in representation. In other words it is the MAP estimation of the true coefficient with this prior that we have the most possible sparsity in representation. Figure 1 states a procedure for solution of such a problem[13]. Once the coefficients estimated we can reconstruct the $\mathbf{c}_{1}$ by $\mathbf{A} \hat{\boldsymbol{\alpha}}$. Similar situation is hold for the second part of the image $\mathbf{c}_{2}$. Also, TV correction is done on the $\mathbf{c}_{1}$ to satisfy the piece-wise assumption on $\mathbf{c}_{1}$. This can be done by simple shrinkage method over $\mathbf{c}_{1}$ by transforming the resultant image to Haar wavelets and apply the soft thresholding of shrinkage and then converting to image space with inverse Haar transform $[9,11]$. Then this overall procedure will be repeated in a way that error energy remained in $\mathbf{R}$ reduced till satisfactory level. Figure 2 depicts all this procedure in a routine based algorithm.

\section{EXPERIMENTAL RESULTS}

In this section, we apply the stated algorithm of Fig. 2 for the reconstruction of gray level still images where some parts are missing. The original image is multiplied with a binary mask of $\mathbf{M}$ and the main aim is to fill out properly the missing parts of an image behind this mask. The corrupted available image is to decomposed in two images called texture and cartoon and try to fill out each layer independently. All the information available is that the location of the missing samples in the original image is known and the components of the image can be represented sparsely over their known dictionaries. Our approach in this work is to choose two known transforms, one to represent the texture and the other for the cartoon. With regards to the actual choice, for the cartoon representation, we used curvelet transform and for the texture; we used local-DCT transform. These dictionaries are nice choice of transform according to our experience dependent on this problem. Maybe that is because of the oscillatory behavior of the local-DCT and the 


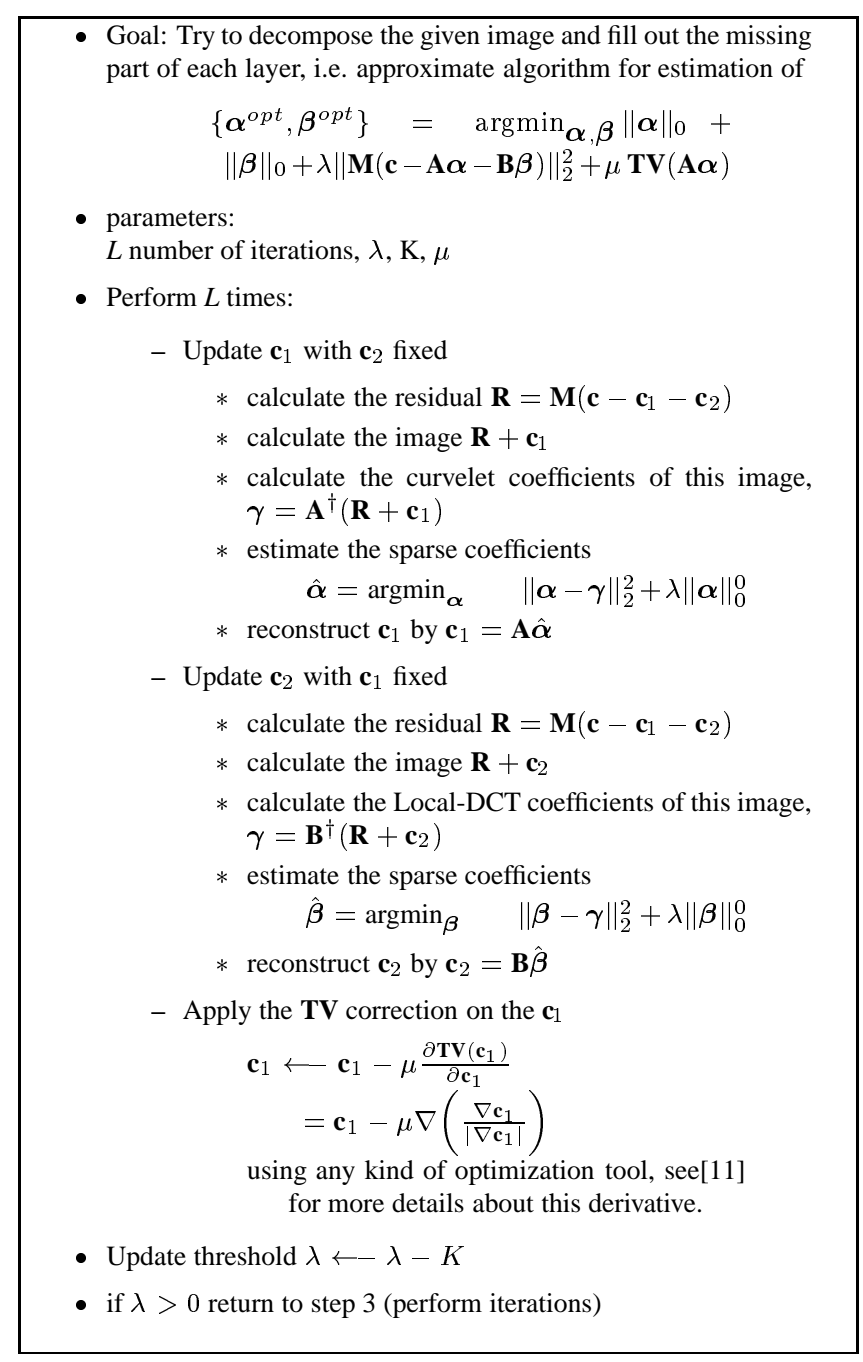

Fig. 2. The final Inpainting algorithm.

multiresolution behavior of curvelets. We must remind that the type of sparse transformation may vary from one image to another [8] but must be mutually independent. Figure 2, illustrates the representation result of decomposition of the Barbara image in to Texture and cartoon when no missing samples are present. In other words, we used identity matrix for $\mathbf{M}$ and only decomposition to cartoon and texture was intended. Similar to the work presented in [9] curvelet transform with six resolution levels is used for the dictionary assigned for the texture and 50\% overlapping discrete cosine transform with a block size of $32 \times 32$ for the dictionary assigned for the cartoon layer. Resolution levels in curvelet and optimal block size in local-DCT transformation were obtained experimentally. Fixed number of iteration $N=100$ is used. Also $\lambda=1$ and $\mu=2$ was the other parameters used.

In Fig. 3, (Top left) we show the original Barbara image, on top right the target regions are masked in white. Region

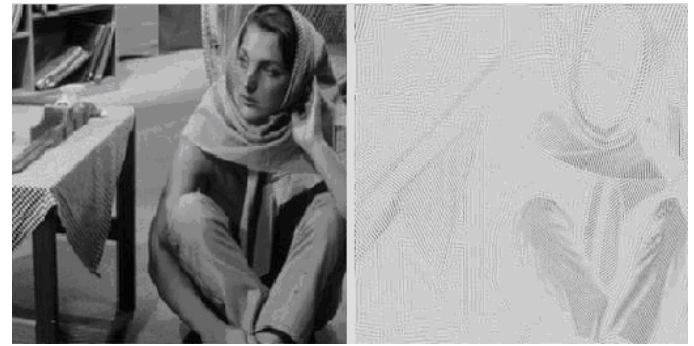

Fig. 3. The representation result in last iteration of proposed algorithm for the Barbara image. (left) Output of curvelet transform with six resolution levels which corresponds to $\mathbf{A s}{ }_{1}^{o p t}$. (right) Output of local-DCT representation with a block size $32 \times 32$ which corresponds to $\mathbf{B s}_{2}^{\text {opt }}$.

filling via our inpainting method using curvelet and localDCT dictionaries are illustrated on bottom left. The result of our algorithm around Barbara's eyes shows no trace of the original holes, and seems natural on Bottom right. Several other experiments are done to examine the validity of the approach mentioned. In order to have a numerical comparison for validity of the algorithm mentioned we have tried different mask with variable size and random positions. Table 1 shows the signal to noise ratio comparison to compare the proposed algorithm with Morphological Component analysis (MCA) presented in [9]. In all these experiments we generated a mask of different size relative to original image size to demonstrate the effect of percentage of missing samples on the performance of reconstruction. It is obvious that when the overall percentage missing parts increases the performance of the proposed algorithm decreases. In comparison presented $P S N R$ for images of size $256 \times 256$ is defined as:

$$
\begin{gathered}
M S E=1 /\left(255^{2}\right)\left(\sum_{i, j=0}^{255} \sum_{i, j=0}^{255}\left(\|\mathbf{c}(i, j)-\hat{\mathbf{c}}(i, j)\|^{2}\right)\right) \\
P S N R=10 \log \left(255^{2} / M S E\right)
\end{gathered}
$$

\section{CONCLUSIONS}

In this paper we presented a novel approach for decomposition of a given signal over two overcomplete dictionaries with special attention in inpainting. It is basically on the basis of decomposition of an image to texture and cartoon layers via sparse combinations of atoms of predetermined dictionaries. The stated algorithm with consideration of totalvariation regularization attempts to fill in the holes in each layer separately and superimposes these layers as a final solution. Experimental results show the efficiency of proposed algorithm in finding the missing samples. Future theoretical work on the general behavior of this algorithm along with 
Table 1. Summary of inpainting PSNR results. In each column the above is corresponding to our approach and the bottom is corresponding to the MCA after 100 iterations. the bold one corresponds with better response.

\begin{tabular}{|c||c||c||c||c||c||c|}
\hline \hline \% of missing samples & LENA & BARBARA & BOAT & Fgrpt $^{1}$ & House & Peppers \\
\hline \hline 2 & $\mathbf{9 5 . 0 0}$ & $\mathbf{8 9 . 6 7}$ & $\mathbf{9 3 . 4 2}$ & $\mathbf{6 2 . 1 1}$ & $\mathbf{9 4 . 7 4}$ & $\mathbf{9 3 . 7 3}$ \\
& 90.42 & 80.38 & 92.17 & 61.85 & 92.23 & 90.15 \\
\hline \hline 5 & $\mathbf{9 3 . 0 6}$ & $\mathbf{7 9 . 3 1}$ & $\mathbf{8 6 . 3 7}$ & $\mathbf{5 6 . 5 6}$ & $\mathbf{9 3 . 7 3}$ & $\mathbf{8 7 . 7 1}$ \\
& 85.81 & 78.41 & 86.08 & 56.71 & 89.95 & 83.01 \\
\hline \hline 10 & $\mathbf{9 0 . 4 7}$ & $\mathbf{7 5 . 2 3}$ & $\mathbf{8 3 . 6 2}$ & $\mathbf{5 2 . 9 3}$ & $\mathbf{8 9 . 8 2}$ & $\mathbf{8 4 . 2 8}$ \\
& 83.32 & 73.51 & 79.62 & 49.13 & 85.60 & 79.53 \\
\hline \hline 15 & $\mathbf{8 5 . 7 5}$ & $\mathbf{7 3 . 9 2}$ & $\mathbf{7 9 . 7 3}$ & $\mathbf{5 0 . 5 6}$ & $\mathbf{8 4 . 3 2}$ & $\mathbf{7 2 . 1 2}$ \\
& 81.87 & 73.11 & 75.13 & 48.12 & 81.40 & 69.27 \\
\hline \hline 20 & $\mathbf{8 0 . 3 8}$ & $\mathbf{6 9 . 4 7}$ & $\mathbf{7 0 . 3 6}$ & $\mathbf{4 9 . 7 4}$ & $\mathbf{7 3 . 2 0}$ & $\mathbf{7 0 . 8 0}$ \\
& 75.05 & 67.53 & 61.29 & 43.92 & 69.29 & 61.58 \\
\hline \hline 25 & $\mathbf{7 0 . 3 2}$ & $\mathbf{6 3 . 5 0}$ & $\mathbf{6 5 . 2 8}$ & $\mathbf{4 8 . 6 2}$ & $\mathbf{6 2 . 1 5}$ & $\mathbf{6 2 . 7 3}$ \\
& 69.28 & 62.29 & 59.46 & 41.90 & 50.19 & 53.81 \\
\hline \hline 30 & 57.19 & 57.41 & $\mathbf{5 8 . 9 5}$ & $\mathbf{4 0 . 2 4}$ & $\mathbf{5 2 . 9 5}$ & $\mathbf{5 5 . 4 3}$ \\
& $\mathbf{6 0 . 1 2}$ & $\mathbf{6 0 . 7 9}$ & 53.30 & 36.43 & 48.61 & 47.17 \\
\hline \hline 35 & 53.04 & 52.71 & 49.98 & 35.79 & $\mathbf{4 9 . 2 6}$ & 38.19 \\
& $\mathbf{5 9 . 3 1}$ & $\mathbf{5 4 . 2 5}$ & $\mathbf{5 0 . 0 3}$ & $\mathbf{3 5 . 8 9}$ & 36.83 & $\mathbf{4 8 . 1 9}$ \\
\hline
\end{tabular}

learning of dictionaries through examples adapted to each layers are two further topics in our current research agenda.

\section{REFERENCES}

[1] S. S. Chen, D.L. Donoho, M.A. Saunder "Atomic decomposition by basis pursuit" SIAM J. Sci. Comput., 20, pp. 33-61, 1994.

[2] J. S. De Bonet "Multiresolution sampling procedure for analysis and synthesis of texture images." Proceedings of SIGGRAPH, 1997.

[3] A. A. Efros, T.K. Leung, "Texture synthesis by nonparametric sampling." IEEE International Conference on Computer Vision, Corfu, Greece, pp. 10331038, September 1999.

[4] V. Caselles, M. Bertalmio, G. Sapiro, C. Ballester, "Image inpainting." Comput. Graph. (SIGGRAPH 2000), pp. 417-424, July 2000.

[5] M. Bertalmio, L. Vese, G. Sapiro, S. Osher, "Simultaneous structure and texture image inpainting." IEEE Trans. Image Process., 12, pp. 882-889, 2003.

[6] L. Vese, S. Osher, "Modeling textures with total variation minimization and oscillating patterns in image processing." Journal of Scientific Computing., vol. 19, pp. 553-577, 2003.
[7] J. Aujol, G. Aubert, L. Blanc-Feraud, A. Chambolle, "Image decomposition: Application to textured images and SAR images" Tech. Rep. ISRN I3S/RR-2003-01-FR, INRIA - Project ARIANA, Sophia Antipolis, 2003.

[8] J. L. Starck, M. Elad, D.L. Donoho, "Image decomposition via the combination of sparse representations and a variational approach." IEEE Trans. Image Process., Vol 14, No 10, pp. 1570-1582, oct. 2005.

[9] M. Elad , J. L. Starck, P. Querre, D.L. Donoho, "Simultaneous cartoon and texture image inpainting using morphological component analysis (MCA)." Journal on Applied and Computational Harmonic Analysis., Vol. 19, pp. 340-358, November 2005.

[10] H. Mohimani, M. Babaei-Zadeh, C. Jutten, "A Fast approach for overcomplete sparse decomposition based on smoothed L0 norm" IEEE Transactions on Signal Processing , Vol. 57, No. 1, January 2009, pp. 289-301.

[11] L. I. Rudin, S. Osher, E. Fatemi "Nonlinear total variation noise removal algorithm", Physica D 60, pp. 259-268, 1992.

[12] A. G. Bruce, S. Sardy, T. Tseng "Block coordinate relaxation methods for nonparametric signal denois- 


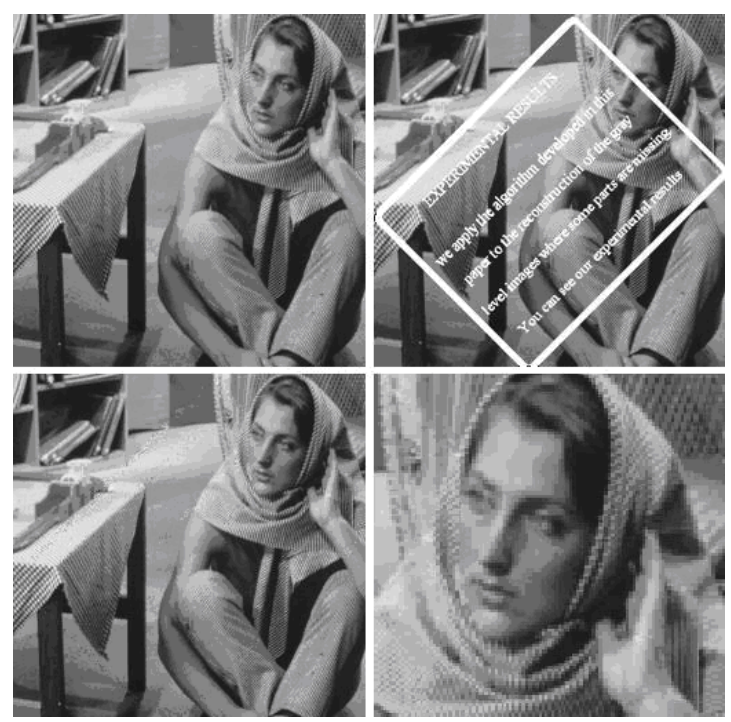

Fig. 4. The reconstruction of the masked image. (Top left) Original image. (Top right) The target regions are masked in white. (Bottom left) Region filling via the proposed inpainting algorithm. (Bottom right) The result of our algorithm around Barbara's eyes.

ing", Proceedings of the SPIE, vol 3391, pp. 75-86, 1998.

[13] S. M. Valiollahzadeh, H. Firouzi, M. Babaie-Zadeh, C. Jutten "Image Denoising Using Sparse Representations.", Proceedings of the ICA2009, pp. 557-564, 2009. 